\title{
UTILITY OF NOVEL OXIDATIVE STRESS BIOMARKERS IN DIAGNOSIS AND PROGNOSIS OD SEPSIS-ASSOCIATED ACUTE KIDNEY INJURY
}

\section{Background}

- Oxidative stress has a pivotal role in the pathophysiology of sepsis-associated acute kidney injury (SA-AKI) (1).

- Established biomarkers of oxidative stress are unstable and difficult to measure in real clinical settings.

- Total oxidant status (TOS), total antioxidant status (TAS) and oxidative stress index (OSI) are emerging biomarkers of oxidative stress.

- These biomarkers were not yet explored in SA-AKI .

\section{Goals}

- To investigate the utility of TOS, TAS and OSI as diagnostic and prognostic biomarkers od SA-AKI in major abdominal surgical patients with sepsis.

\section{Materials and Methods}

- Prospective, observational study.

- 53 major abdominal surgical patients with sepsis divided into SA-AKI $(n=37)$ and non-SA-AKI $(n=16)$ group were compared to 50 controls without sepsis matched by age, gender, comorbidities and type of surgery.

- Blood and urine samples from the septic patients were collected on admission to Intensive Care Unit (ICU) and 24, 48, 72 and $96 \mathrm{~h}$ later and once from the controls.

- Levels of TOS and TAS were measured using novel automated colorimetric method developed by Erel $(2,3)$.

- OSI was calculated as TOS to TAS percent ratio (4).

- Urea, creatinine and neutrophil gelatinase-associated lipocalin (NGAL) were also measured.

- American Society of Anaesthesiology (ASA), Sequential Organ Failure Assessment (SOFA) and Acute Physiology and Chronic Health Evaluation II (APACHE II) scores were calculated.

\section{Results}

Table 1. Baseline characteristics of the patients with sepsis and the control group
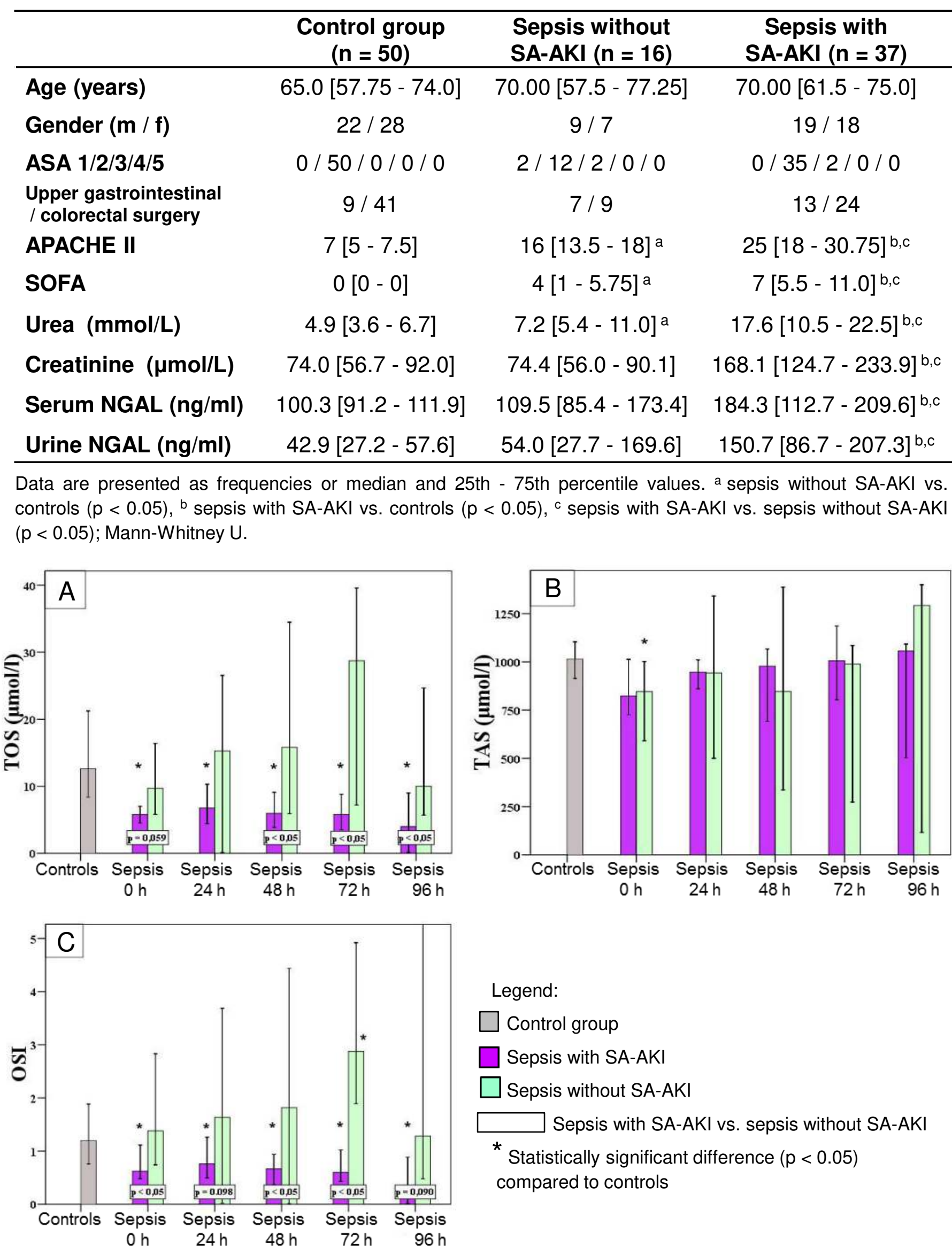

Legend:

$\square$ Control group

$\square$ Sepsis with SA-AKI

$\square$ Sepsis without SA-AKI

$\square$ Sepsis with SA-AKI vs. sepsis without SA-AKI

${ }^{*}$ Statistically significant difference $(p<0.05)$

compared to controls

Figure 1. Dynamics of (A) TOS, (B) TAS and (C) OSI in patients with sepsis with comparison to control group

Data are expressed as minimum, maximum, median, and interquartile range. Mann-Whitney $\mathrm{U}$ test.

- Septic patients with SA-AKI had lower TOS and OSI but similar TAS compared to septic patients without SA-AKI during $96 \mathrm{~h}$ following admission to ICU (linear mixed models: PTOS $=0.006$, $\mathrm{POSI}=0.001$, PTAS $=0.070$ ).

- TAS, TOS and OSI were indirectly correlated to urea, creatinine, NGAL, SOFA and APACHE II score.

.Table 2. TOS, TAS and OSI as diagnostic biomarkers of SA-AKI

\begin{tabular}{cc}
\hline & AUC-ROC $(95 \%$ C. I. $)$ \\
\hline TOS & $0.732(0.564-0.901){ }^{*}$ \\
TAS & $0.666(0.495-0.837)$ \\
OSI & $0.720(0.547-0.893){ }^{*}$ \\
\hline${ }^{*} \mathrm{p}<0.05$ &
\end{tabular}

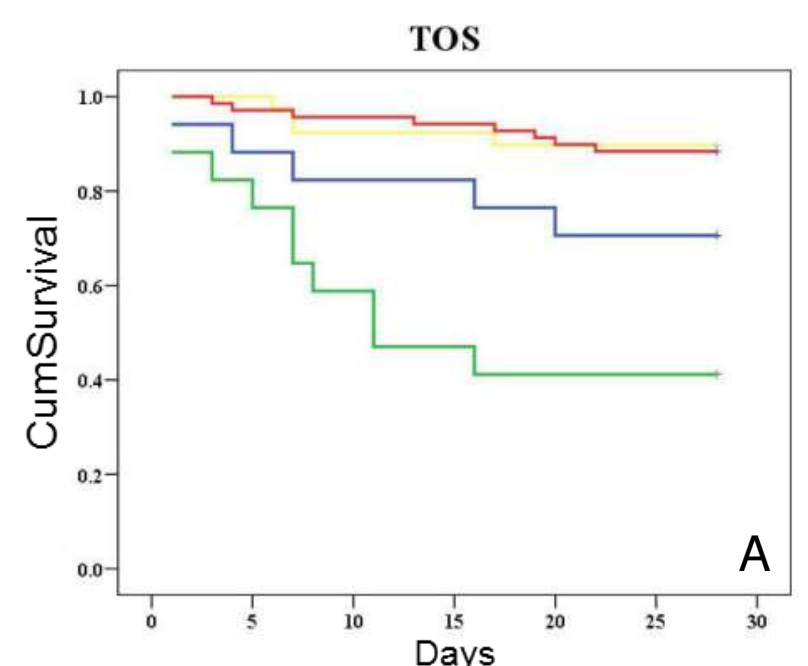

os

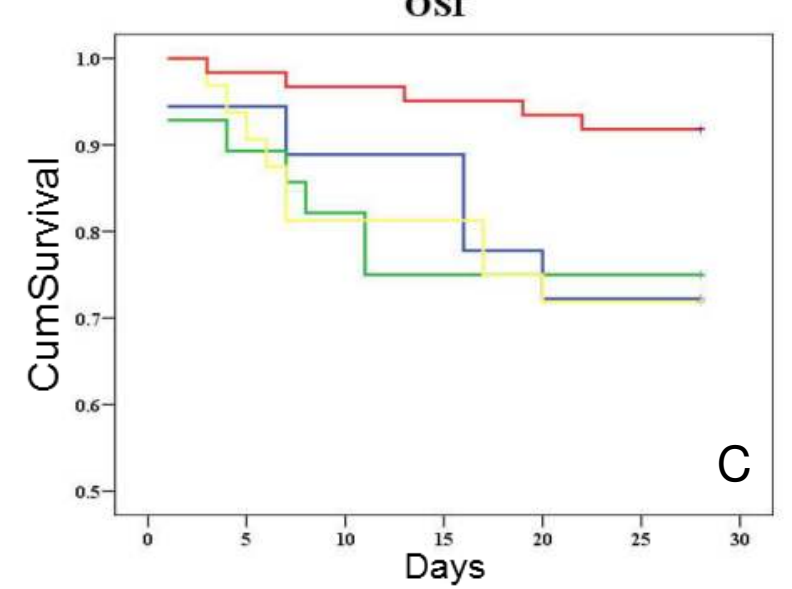

Figure 2.TOS, TAS and OSI as prognostic biomarkers of sepsis Patients are stratified in quartiles. Kaplan-Meier estimates of 28 day survival. Log rank: (A) $P_{\text {TOs }}<0.001$ (B) $\mathrm{P}_{\text {TAS }}=0.918$ and (C) $\mathrm{P}_{\mathrm{OSI}}=0.045$

\section{Conclusions}

- SA-AKI was associated with lower TOS and OSI.

- TOS and OSI were, unlike TAS, good diagnostic and excellent prognostic biomarkers of SA-AKI in major abdominal surgical patients with sepsis.

\section{References}

Gomez H, Ince C, De Backer D, Pickkers P, Payen D, Hotchkiss J, et al. A unified theory of sepsis-induced acute kidney injury: inflammation, microcirculatory dysfunction, bioenergetics, and the tubular cell adaptation to injury. Shock. 2014 Jan;41(1):3-11. Erel 0 . A new automated colorimetric

med for measuring total oxidant status. Clinical biochemistry. 2005

atomated method to measure total antioxidant response against potent free radical reactions. Clinical biochemistry. $2004 \mathrm{Feb} ; 37(2): 112-9$.

Sirmatel O, Sert C, Sirmatel F, Selek S, Yokus B. Total antioxidant capacity, total oxidant status and oxidative stress index in the men exposed to $1.5 \mathrm{~T}$ static magnetic field. General physiology and biophysics. 2007 Jun;26(2):86-90 